\title{
Dynamics of the super pinch electron beam and fusion energy perspective
}

\author{
D. R. Welch® and D. V. Rose $\odot$ \\ Voss Scientific, LLC, Albuquerque, New Mexico 87108, USA \\ D. V. Novikov®, M. E. Weller@, and A. A. Esaulov \\ Proton Scientific Technologies, Inc., Oak Ridge, Tennessee 37830, USA \\ G. H. Miley \\ University of Illinois, Urbana, Illinois 61801, USA
}

(Received 26 July 2021; accepted 22 November 2021; published 16 December 2021)

\begin{abstract}
The characterization of a high current, relativistic electron beam, designated the Super Pinch electron beam, has been performed using compact pulsed-power accelerators of various architectures with a novel diode geometry. The Thunderbird accelerator has an initial $1.5-\mu$ s rise time and $150-\mathrm{kA}$ peak current. The drive voltage is compressed to produce a $12-\mathrm{ns} 500-\mathrm{kV}$ voltage pulse generating a $40-\mathrm{kA}$ electron beam, which apparently exceeds the Alvén current limit although the useful current at small radius is an order of magnitude less. Using an insulated hollow cathode with a $0.5-\mathrm{cm}$ anode-cathode gap, the large current is enabled by the evolution of plasma from the dielectric sleeve enveloping the cathode and a 0.5 -mm wire anode. Post-shot recovery of the anode target and measurements of its deformation and damage allow an evaluation of the enhanced electron-beam focusing whereby significant beam energy is delivered to the record small, microscopic volume inside the anode target. The electron beam is seen to have conditions favorable to those needed to ignite compressed fuel in inertial confinement fusion. These results motivated a deeper insight using theoretical modeling with hybrid particle-in-cell codes. The modeling presented in this paper shows an electron beam radius of $<10 \mu \mathrm{m}$ containing a current of 3-6 kA or $>500 \mathrm{MA} / \mathrm{cm}^{2}$ current density. Scaling up the accelerator, such preferred focusing, target penetration, and affordability of the pulsed power generated electron beams open a new opportunity for the application of the mainstream pulsed power devices in the research and development of fusion energy.
\end{abstract}

DOI: 10.1103/PhysRevAccelBeams.24.120401

\section{INTRODUCTION}

In inertial confinement fusion (ICF), hydrogen fuel is compressed to sufficient densities and temperatures to ignite. The 500-keV, 50-kA Thunderbird electron beam accelerator [1] produces a high-intensity electron beam onto a wire anode using an insulated hollow cathode. Although the root-mean-square electron beam radius is an order of magnitude larger, the core on axis beam is roughly $10 \mu \mathrm{m}$ in radius exhibiting extreme intensities, $\sim 10^{14} \mathrm{~W} / \mathrm{cm}^{2}$, an intensity heretofore obtained only in laser foci. This super pinch diode (SPD) diode operates with a rapidly falling impedance similar to a plasma-filled self-magnetic-pinch (SMP) diode, [2,3,4,5,6] Both the SMP and SPD diodes exhibit rapid plasma motion, bipolar operation, and falling impedance, but due to the cathode

Published by the American Physical Society under the terms of the Creative Commons Attribution 4.0 International license. Further distribution of this work must maintain attribution to the author(s) and the published article's title, journal citation, and DOI. and anode geometries, the SPD acts as a cool ChildLangmuir (CL) device while the typical SMP diode operates in equilibrium as a current-limited device $[7,8]$. The Thunderbird accelerator, making use of a plasma opening switch (POS), maintains a $240-300-\mathrm{kV}$ diode voltage for over $10 \mathrm{~ns}$ even as the impedance falls an order of magnitude to $<10 \Omega$. [1] Post-mortem analysis of the anode wire suggests that the intense beam is actually boring a hole into the solid wire over 1-2-mm deep. The inferred intensities of the beam make it a good candidate for heating and igniting compressed fuel for ICF.

The diode operation relies on the evolution of plasmas at small radius. This evolution is controlled by the addition of a plastic sleeve on the cathode with a hole in the center. Thus, cathode side plasmas evolving largely from the inner sleeve are constrained to the hole emerging as a jet of plasma toward the anode wire. Similarly, the current path must connect from the exposed metal within the cathode hole before being conducted through the plasma at small radius toward the anode. Electrons reaching the front of the expanding cathode plasma emit in space-charge-limited fashion into the shrinking gap. After the plasma has largely 
traversed the entire gap, the electrons are accelerated across a small gap at the anode wire resulting in a falling impedance. The hybrid particle-in-cell codes Lsp and Chicago have been used extensively to describe the operation of high-current diodes with evolving plasmas. $[9,10]$ In calculations presented here, the Chicago code is used to model the Thunderbird accelerator including the pulsed-power circuit driving the SPD. The diode simulations are highly resolved $(5-\mu \mathrm{m}$ radial and $10-\mu \mathrm{m}$ axial resolution) in two dimensions to characterize the highintensity electron beam as it strikes the anode wire. Simulated diode currents are in good agreement with measurements from B-dot monitors [1]. Subsequent hybrid simulations of the characterized relativistic electron beam entering a $\mathrm{Cu}$-wire anode show qualitatively the hole boring seen in experiment.

The intense electron beam produced with the SPD has potential applications to plasma heating and fusion energy $[11,12,13,14,15]$. The beam transport from the diode through a solid wire to compressed fusion fuel such as DT is one advantage of the SPD over other pulse power diodes. While the physics of the beam interaction with dense fuel requires significant investigation, the requirements for fusion energy suggest beam ignition of compressed fuel is possible.

In this paper, we present results from simulations of the SPD mounted on the Thunderbird accelerator that assess key elements of the diode including impedance evolution due to contaminant plasma motion from the electrodes. We characterize the electron beam at the anode wire and study the propagation of the beam into a solid copper anode in a separate simulation. We present the details of the Thunderbird equivalent circuit and simulation models in Sec. II. Simulation results of the diode are presented and compared with data in Sec. III. Hole boring simulations of the beam within the copper anode are presented in Sec. IV. We discuss the relevance of the SPD electron beam to fusion energy in Sec. V and present conclusions in Sec. VI.

\section{ELEMENTS OF THE THUNDERBIRD ACCELERATOR}

The Thunderbird accelerator, as shown in Fig. 1, is driven by a fast voltage pulse compressed by a POS. The fast pulse drives a peak $130-\mathrm{kA}$ current and $500-\mathrm{kV}$ voltage into the SPD which we now describe.

\section{A. Diode operation}

The diode operates in a similar fashion as a plasma-filled SMP diode with rapidly falling impedance due to plasma evolution from the cathode and anode. Tightly coupled experimental and theoretical work has greatly improved the understanding of diode performance, in particular its electrical parameters and plasma dynamics [16]. Shown schematically in Fig. 2, the evolution of the SPD has three distinct phases: plasma formation, plasma expansion with shrinking electrical gap, and dense plasma enveloping the anode with rapid diode impedance fall, detailed below. The high-intensity electron beam is the result of suppressing the cathode current at large radius off the outer radius of the cathode. This is achieved with the thick plastic sleeve through which current flow is inhibited and the hole in the center uncovering the cathode (nominally $1 \mathrm{~mm}$ radius) from which nearly all the current flows. The electrode materials are typically copper. The anode wire is nominally $0.5 \mathrm{~mm}$ in radius. In phase 1 as the electric field stress rises, plasma forms on the exposed cathode and plastic surface as it breaks down with $>20-\Omega$ diode impedance. Current can only connect to the cathode at the small-radius exposed cathode. The dielectric plasma connects to that of the cathode as it expands inward and axially toward the anode

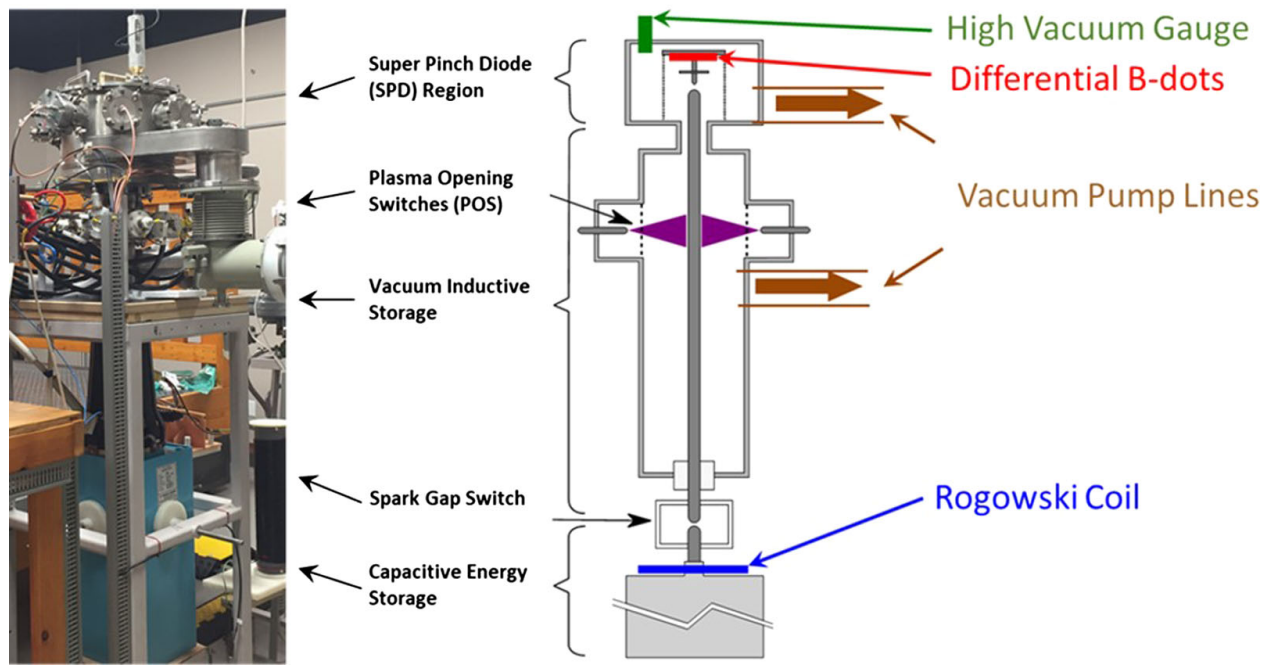

FIG. 1. A picture (left) and schematic (right) of the Thunderbird electron beam accelerator. 


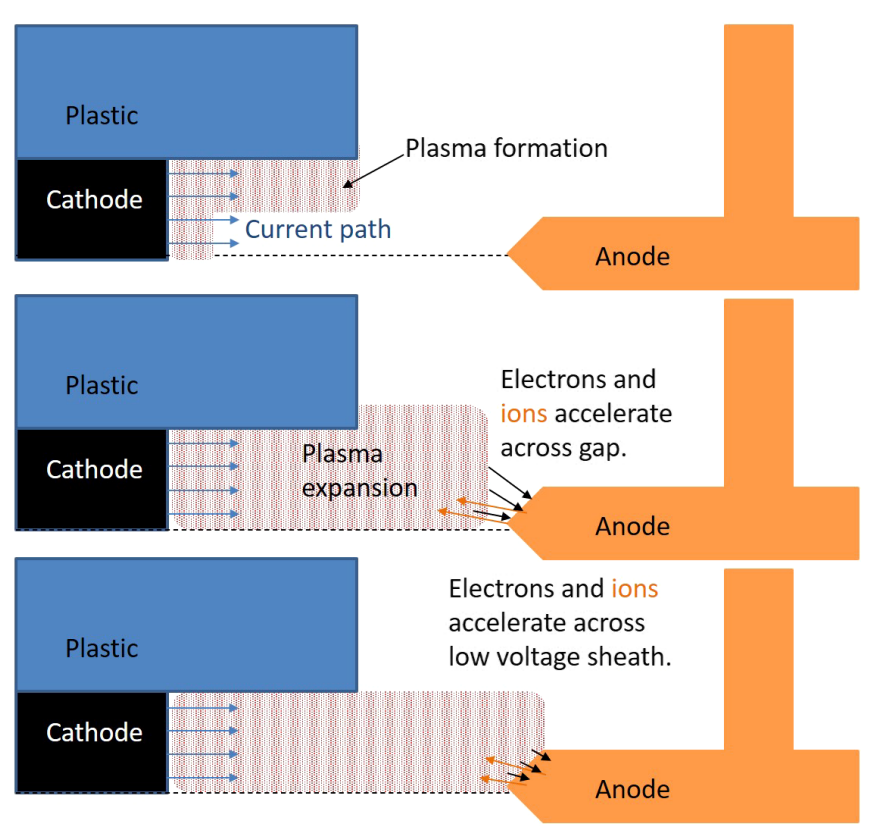

FIG. 2. Three phases of evolution of the Super Pinch Diode are shown: (1) explosive cathode emission, flashover of the plastic cathode sleeve, plasma formation with high impedance $>20 \Omega$ (top plot); (2) shrinking electrical gap from plasma motion with moderate impedance 5-20 $\Omega$ and small beam spot (middle plot); and rapidly falling impedance and plasma envelopes the anode (bottom plot). An electron beam and counter-flowing ion beam are produced across the sheath near the anode. Lower impedance operation occurs as the sheath gap shrinks due to plasma motion and more of the anode is covered by cathode plasma.

wire. The effective AK or electrical gap is thus reduced until, in phase 2 , producing moderate diode impedance (5-20 $\Omega$ ) and a small electron beam spot. A small fraction of the total current from the anode is carried by ions as well. In phase 3, the plasma reaches and envelopes the anode wire rapidly decreasing the diode impedance $(<5 \Omega)$ with the sheath becomes very small.

We have found in simulations that the typical experimental operating pressure range of $10-50 \mu$ Torr optimizes the pinch producing the highest intensities at the anode. The surface contaminant inventory and, thus, plasma supply are limited at reduced vacuum pressure below $1 \mu$ Torr causing the electron beam to be accelerated across the diode at larger radius with moderate impedance. Lower impedance and possible degradation of super pinch occurs as cathode plasma envelops anode. The voltage falls in a small sheath between the cathode plasma and the anode. The beam is thus accelerated across a small distance suppressing any three-dimensional instabilities. It is important to note that experiments have been performed without the use of the plastic sleeve that produce no evidence of a high intensity and focused electron beam. Similarly, in experiments carried out without the use of the compressed current (see Ref. [1] for uncompressed current shots), i.e., $>1 \mu \mathrm{s}$ rise time, there was also no evidence of a high intensity and focused electron beam. Therefore, two critical components of the super pinch electron beam are the presence of the plastic sleeve and a compressed pulse (likely $<50 \mathrm{~ns}$ ).

\section{B. Surface physics model}

In the SPD region, there are initially no free current carriers and the pulsed electromagnetic wave increases the applied AK (anode-cathode) voltage and electric field stresses. For typical bare metal and plastic surfaces, electrons are emitted from the cathode above an electric field threshold of roughly $240 \mathrm{kV} / \mathrm{cm}$. As the threshold is exceeded, the surface breaks down explosively yielding a surface plasma. In the electrode model used in the Chicago code [17], the explosive breakdown of the cathode increases the surface temperature by $300{ }^{\circ} \mathrm{C}$ (an adjustable parameter in the model) desorbing surface contaminants. This behavior is assumed for the dielectric as well for the thermal desorption model of plastic. The surface temperatures are also increased from charged particle impact heating. Ohmic heating for the metal surfaces is included but is small until late in time. The Ohmic heating model is described in Ref. [18], where the thermal conductivity and specific heat are assumed to be constant.

After a surface temperature rise of roughly $400{ }^{\circ} \mathrm{C}$, neutral molecules are desorbed thermally [discussed in Ref. [19]], ionize, and then provide a source of electrons or ions for acceleration into the diode AK gap. Here, the neutrals are assumed to be comprised of water molecules which are a significant constituent of surface contaminants. A completely fragmented and ionized water plasma $\left(3 \mathrm{e}^{-}\right.$, $\left.2 \mathrm{H}^{+}, \mathrm{O}^{+}\right)$is produced within a cell of the material surface. The assumption of the model is that the energy required to ionize and dissociate the water is small (roughly $15-20 \mathrm{eV}$ ) compared with the gap voltage (order $1 \mathrm{MV}$ ). Detailed smaller-scale breakdown simulations have shown this assumption leads to a more rapid ionization of the desorbed water but yields a qualitatively similar plasma profile delayed by $\sim 3 \mathrm{~ns}$ [20]. This desorption model uses the Temkin isotherm which is based largely on the experimental results of Li and Dylla [21], and Dylla et al. [22], and the theoretical analysis of La Fontaine [23]. Discussed in detail in Ref. [19], we assume a $10 \mu$ Torr pressure giving one adsorbed monolayer of water (nominally defined here as $8 \times 10^{15}$ molecules $/ \mathrm{cm}^{2}$ ) with an initial $0.83 \mathrm{eV}$ binding energy. In one simulation described in Sec. III ( $\operatorname{sim} 2$ of Table I), we substitute a stimulated desorption model for the plastic surface flashover in which a charged particle impact desorbs a water molecule of the same weight.

\section{Thunderbird circuit}

The Thunderbird circuit used to drive the SPD simulations has been constructed as shown in Fig. 3 . Thunderbird is a compact pulsed power generator with a 
TABLE I. A summary of the Chicago SPD simulations.

\begin{tabular}{lcccc}
\hline \hline Simulation & $\begin{array}{c}\text { Vacuum } \\
\text { pressure } \\
(\mu \text { Torr })\end{array}$ & $\begin{array}{c}\text { Water } \\
\text { coverage } \\
\left(10^{15} \mathrm{~cm}^{-2}\right)\end{array}$ & $\begin{array}{c}\text { Plastic } \\
\text { desorption } \\
\text { model }\end{array}$ & $\begin{array}{c}\text { Beam power } \\
\text { density at anode } \\
\left(\mathrm{TW} / \mathrm{cm}^{2}\right)\end{array}$ \\
\hline sim1 & 10 & 8 & Thermal & 180 \\
$\operatorname{sim} 2$ & 10 & 8 & Stimulated & 130 \\
$\operatorname{sim} 3$ & 20 & 16 & Thermal & 80 \\
$\operatorname{sim} 4$ & 1 & 4 & Thermal & 45 \\
\hline \hline
\end{tabular}

25 to $35 \mathrm{~ns}$ current rise time to a peak current of $\sim 130 \sim \mathrm{kA}$ [1]. Here a POS, indicated in the figure, provides pulse compression. The discharging capacitor current is shunted by the conductive plasma for $925 \mathrm{~ns}$. The plasma resistance then increases within a nanosecond timescale releasing the accumulated magnetic flux to the load region. The POS plasma is provided by 12 plasma guns (PGs) (see Ref. [1] and references within). The various inductive, capacitive and resistive elements relate as follows:

$L_{s}=L_{0}+L_{1}=240 \mathrm{nH}$,

$L_{2}=75 \mathrm{nH}$,

$L_{3}=L_{5}=20 \mathrm{nH}$,

$C=3000 \mathrm{nF}$,

$R_{0}=0.025 \Omega$,

and,

$R_{p}=0.001+(30-0.001) \frac{t-925 \mathrm{~ns}}{40 \mathrm{~ns}} \Omega \mathrm{t}>925 \mathrm{~ns}$

$0.001 \Omega \mathrm{t}<925 \mathrm{~ns}$.

After $925 \mathrm{~ns}$, the POS plasma resistance $R_{p}$ rises from 0.001 to $30 \Omega$ in $40 \mathrm{~ns}$ accounting for the fast voltage rise. The SPD is included in the circuit as either a circuit element or a finite difference time domain simulation (FDTD) volume. We will compare the FDTD simulated diode resistance with the operation of the circuit including the diode element model which uses an effective diode resistance $R_{D}$ based on the CL current response to voltage $V_{D}(t)$

$$
R_{D}(t)=\frac{9}{4 \pi r_{D}^{2} \varepsilon_{0}} \sqrt{\frac{m_{e}}{2 e}} \frac{\left(g-\left(t-t_{D}\right) u_{g}\right)^{2}}{\sqrt{V_{D}(t)}},
$$

where $d, g$, and $u_{g}$ are the effective emission diameter, initial gap length, and plasma velocity across the gap. The diode begins to conduct current at $t=t_{D}$. Once the gap completely closes the effective gap becomes the Debye sheath size.

The diode model in the circuit, Eq. (2), is now tested against an FDTD description of the diode (simulation sim1 details are discussed in Sec. III). In both cases, we calculate

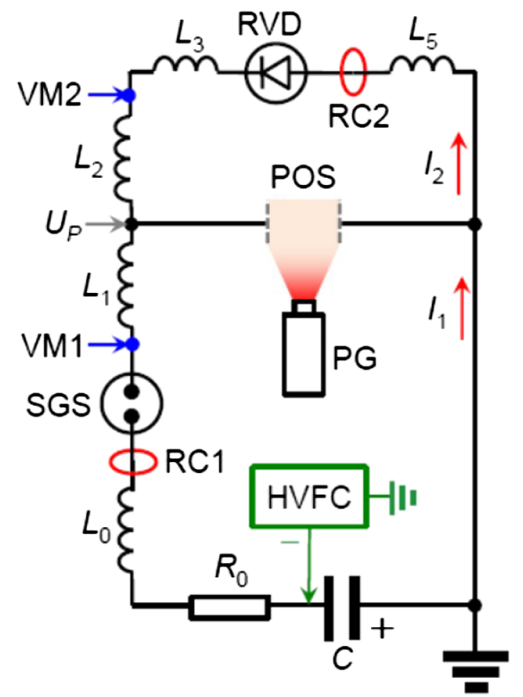

FIG. 3. The details of the Thunderbird circuit attached to the $\mathrm{SPD}$ at the top. The plasma generator (PG) supplies the plasma inventory to the POS.

the voltage and current in the diode. As shown in Fig. 4, the comparison is good indicating the CL emission model with declining gap is a reasonable model for the diode support the physical description in Fig. 2. The current rises throughout the pulse reaching $20 \mathrm{kA}$ at $550-\mathrm{kV}$ peak voltage at $12 \mathrm{~ns}$. The diode impedance falls continuously reaching roughly $5 \Omega$ at $18 \mathrm{~ns}$.
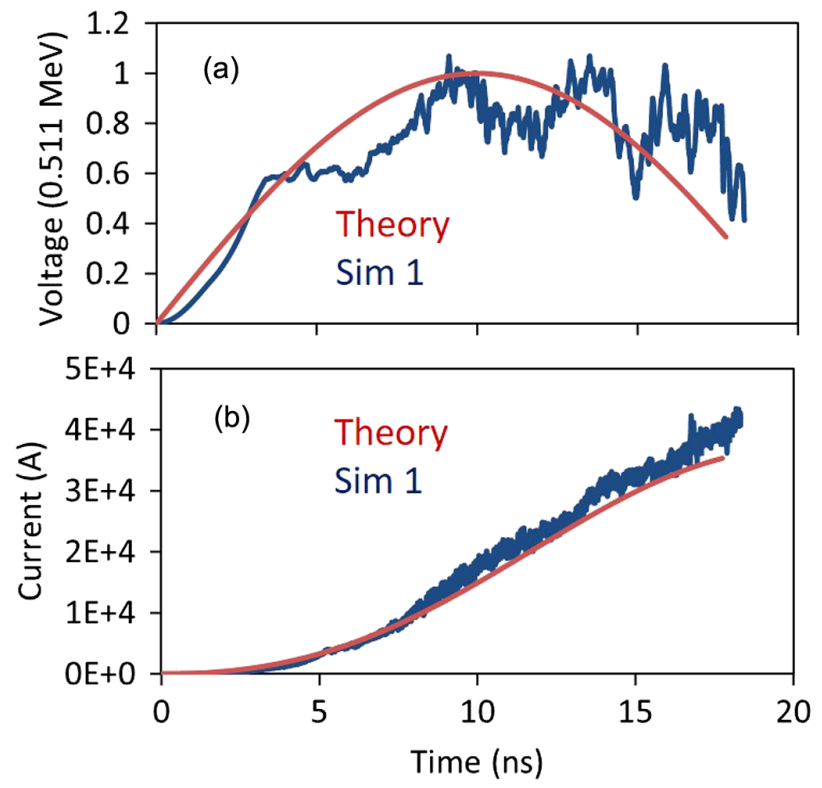

FIG. 4. In (a), the voltage used in the theory and the simulated diode voltage are plotted, in (b) the theoretical and simulated ( $\operatorname{sim} 1$ using only thermal desorption of contaminants) diode currents are plotted. Sim1 assumed an $8 \times 10^{15} \mathrm{~cm}^{-2}$ contaminant areal density. 


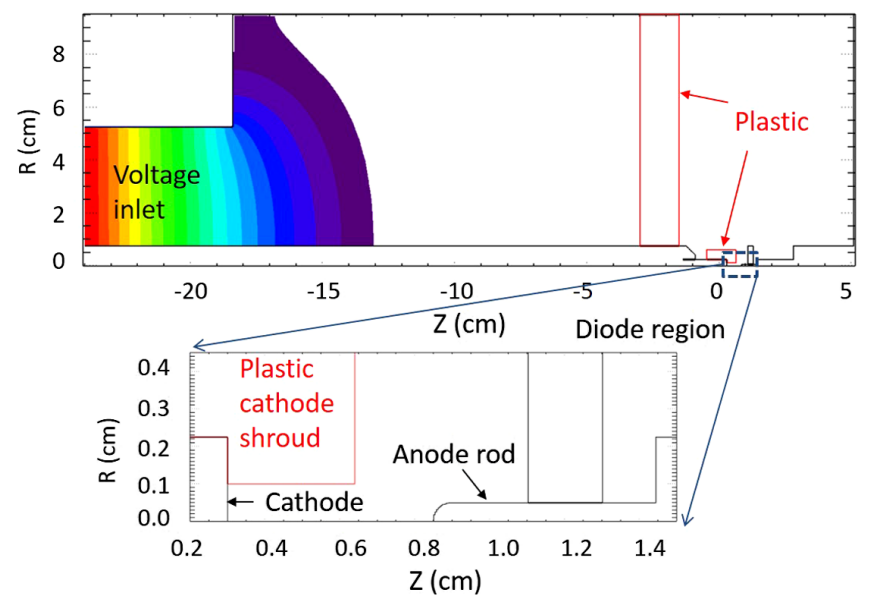

FIG. 5. The computational model of the Thunderbird SPD is shown. The voltage inlet is driven by the Thunderbird circuit. The diode region details are shown in the exploded view. The cathode and anode rod are copper.

\section{SCALING OF THE THUNDERBIRD SPD IMPEDANCE WITH CONTAMINANT COVERAGE}

We have constructed the FDTD representation of the diode with the geometry shown in Fig. 5 and completed a series of two-dimensional simulations of the SPD. The addition of the plastic sleeve was critical to achieving the super pinch behavior. This sleeve changes the operation of the diode from that of a conventional SMP to the SPD. Four frames showing the electron density and enclosed current for a $10-\mu$ Torr pressure in sim $1\left(8 \times 10^{15}\right.$ areal coverage of contaminants on the surfaces using the model discussed in Sec. II B) are shown in Fig. 6. The simulation includes all relevant physics packages in Chicago including spacecharge-limited emission from all surfaces (the cathode and anode are copper) and electrode plasma evolution via a thermal (or stimulated) desorption model, and evolution of water into electrons, $\mathrm{H}^{+}$and $\mathrm{O}^{+}$ions. The simulations use $5-\mu \mathrm{m}$ radial and $10-\mu \mathrm{m}$ axial resolution in the diode and $60 \mathrm{fs}$ temporal resolution. The simulation results do not significantly change at half the spatial and temporal resolution indicating the simulations are converged and accurate.

The plasma evolution enables extremely high-current density. We observe that some electrons are emitted further upstream at larger radius, i.e., outside the cathode hole. As the diode impedance falls, these electrons are quickly retrapped to the cathode and do not carry significant current into the diode region. Thus, most of the current carrying electrons are born at small radius within the sleeve hole. Plasma from inside the plastic hole connects to the bare cathode and flows radially inward and axially forward behaving as a PG. The plasma is shot directly at the anode wire. Thus, the plasma density increases toward the axis enabling more current to flow at very small radius with a

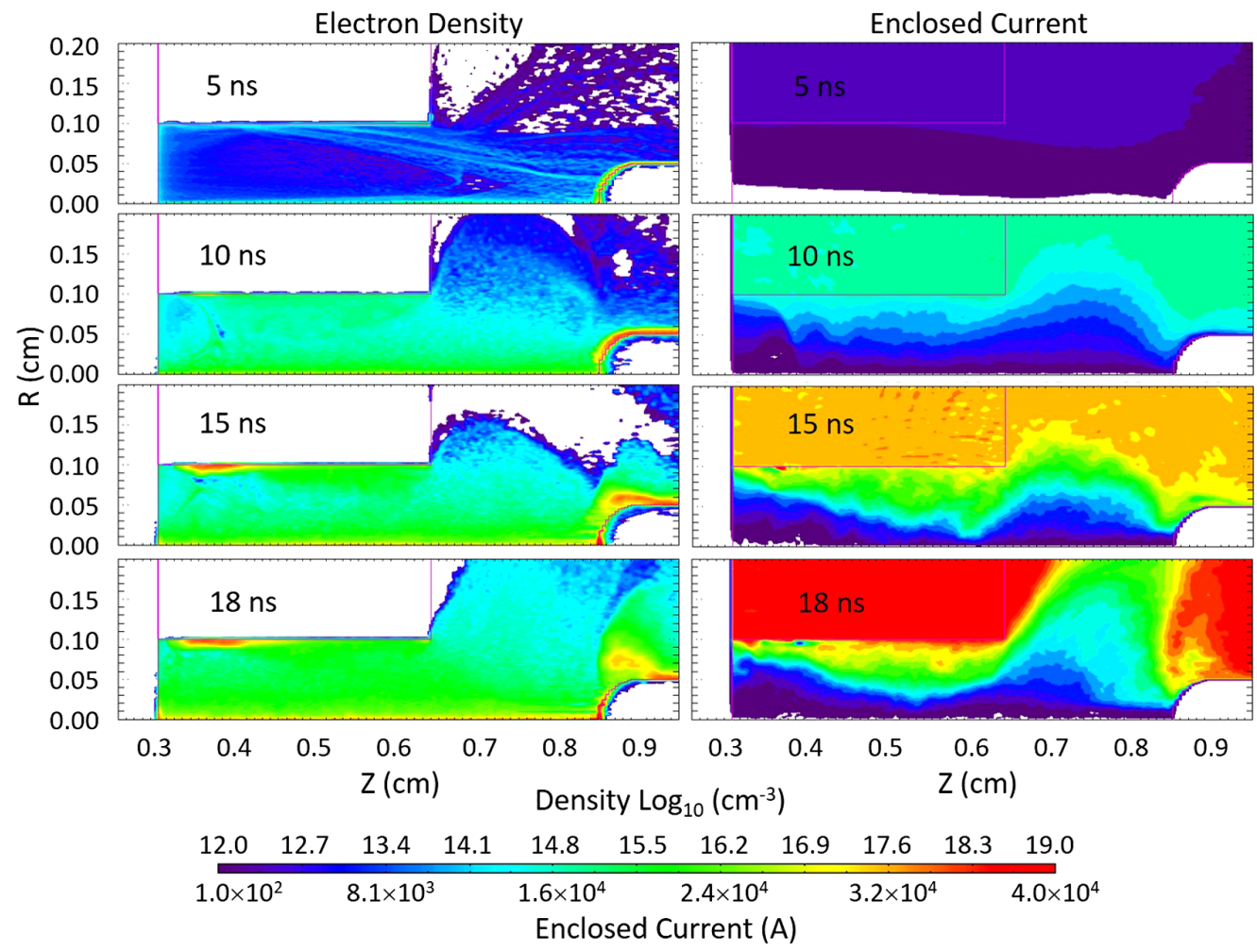

FIG. 6. The electron densities, on the left, after $5 \mathrm{~ns}, 10 \mathrm{~ns}, 15 \mathrm{~ns}$, and $18 \mathrm{~ns}$ are plotted for sim1. The enclosed current (or current within the given radius) is plotted on the right. 
strongly-peaked on axis radial current density distribution. The electrode plasmas significantly reduce the AK gap after roughly $10 \mathrm{~ns}$ and, as predicted by the diode impedance model, rapidly decrease the diode impedance. We find the voltage peaks at about $300 \mathrm{kV}$ in the AK gap region. The enclosed current (or current flowing within a given radius at that $z$ position) plotted in Fig. 6 shows that significant $(>90 \%)$ current flows at a larger radius. The current density falls off roughly linearly with radius near the anode wire with $3 \mathrm{kA}$ flowing within $10-\mu \mathrm{m}$ radius at the wire by $14 \mathrm{~ns}$. The high-plasma density leads to $>900-\mathrm{MA} / \mathrm{cm}^{2}$ current density within $10-\mu \mathrm{m}$ radius.

The plasma evolution off the plastic can be modeled as a pure thermal desorption process due to dielectric breakdown and heating (when the surface electric field $|\mathrm{E}|>240 \mathrm{kV} / \mathrm{cm}$, the plastic surface temperature is increased $300^{\circ} \mathrm{C}$ ) or by a purely stimulated process where electrons and ions impact the surface and desorb the water. The results using the thermal desorption model in sim1 are compared with a stimulated emission model with $10^{-16} \mathrm{~cm}^{2}$ cross section for all charged particles (sim2). Both have an $8 \times 10^{15} \mathrm{~cm}^{-2}$ water coverage. We turn off the thermal desorption from the plastic sleeve entirely in this simulation. Interestingly, the diode impedance behavior is not significantly affected by this drastic change in desorption mechanism as seen in Fig. 7(a) where the impedance in the two simulations is compared with the simple theory. In the stimulated plastic desorption simulation $\operatorname{sim} 2$, the rate of water desorption in the diode was

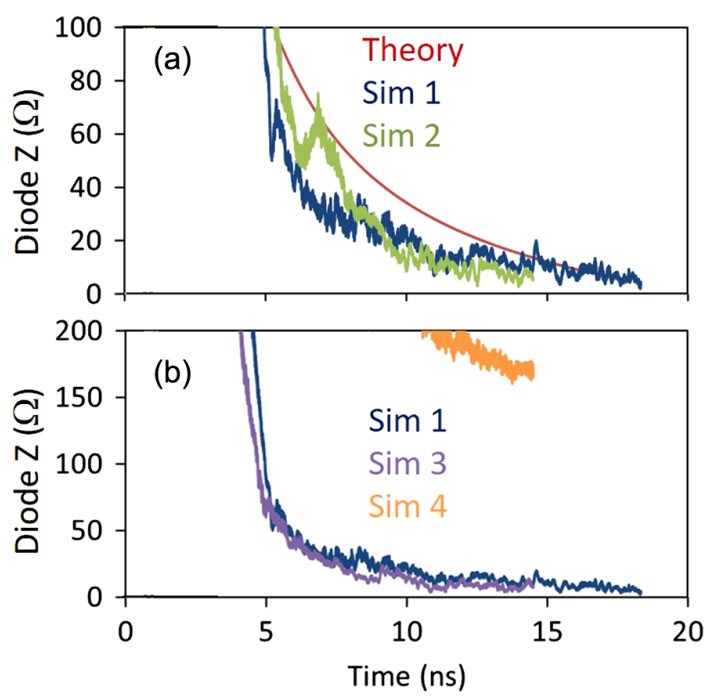

FIG. 7. The diode impedance for the Thunderbird SPD is plotted for various vacuum pressures. (a) the theoretical and simulated (sim 1 using only thermal desorption and $\operatorname{sim} 2$ with only stimulation desorption of contaminants) diode impedances are plotted, and (b), the simulated (sim1 with $8 \times 10^{15} \mathrm{~cm}^{-2}$ contaminants and sim 3 with $16 \times 10^{15}-\mathrm{cm}^{-2}$ contaminants and sim4 with $4 \times 10^{15} \mathrm{~cm}^{-2}$ with thermal desorption) diode impedance are plotted. delayed roughly $1.5 \mathrm{~ns}$ before reaching roughly the same peak. Thus, the stimulated desorption leads to initially a slower impedance fall then accelerates to a smaller value than the thermal case and theory by $14 \mathrm{~ns}$. Thus, the details of the surface desorption are not critical to the basic diode behavior.

The dependence on water coverage is seen in Fig. 7(b) where we compare sim 1 with $16 \times 10^{15} \mathrm{~cm}^{-2}$ in $\operatorname{sim} 3$ and a smaller value of $4 \times 10^{15} \mathrm{~cm}^{-2}$ in sim4. Both $\operatorname{sim} 3$ and sim4 have thermal desorption from the plastic. Sim3 has a more rapid impedance fall to a $30 \%$ smaller value by $14 \mathrm{~ns}$. The higher density of the contaminant plasma enables more current to be conducted. The smallest coverage simulation has a lethargic impedance behavior falling below $200 \Omega$ only after $10 \mathrm{~ns}$. These simulations suggest a pump down pressure $>10 \mu$ Torr is required to achieve the high current density and $>100-\mathrm{TW} / \mathrm{cm}^{2}$ intensity. The electron beam striking the anode in sim 1 had roughly a $<10-\mu \mathrm{m}$ radius at half the current density (containing $3 \mathrm{kA}$ current) and a fairly constant $360 \mathrm{keV}$ energy. In the Sec. IV, we inject this characterized beam into a solid density $\mathrm{Cu}$ anode and study its hole boring.

The falling diode impedance and induced field from rising current limit the voltage across the gap. The highest impedance, shown in Fig. 7, is calculated for the lowest contaminant coverage $\left(4 \times 10^{15} \mathrm{~cm}^{-2}\right.$ water molecules, corresponding to a vacuum pressure of roughly $1 \mu$ Torr). After the plasma has closed the AK gap, electrons are accelerated across the small sheath to the anode. The simulations show that the lower impedance operation gives the highest overall power to the electron beam with power scaling strongly with water coverage (see Table I).

The highest beam intensity is found in the intermediate pressure simulations, however. These results are inferred from current densities and voltages calculated at various times near peak power. For four of the simulations, the peak beam power densities are listed in the Table I. The beam

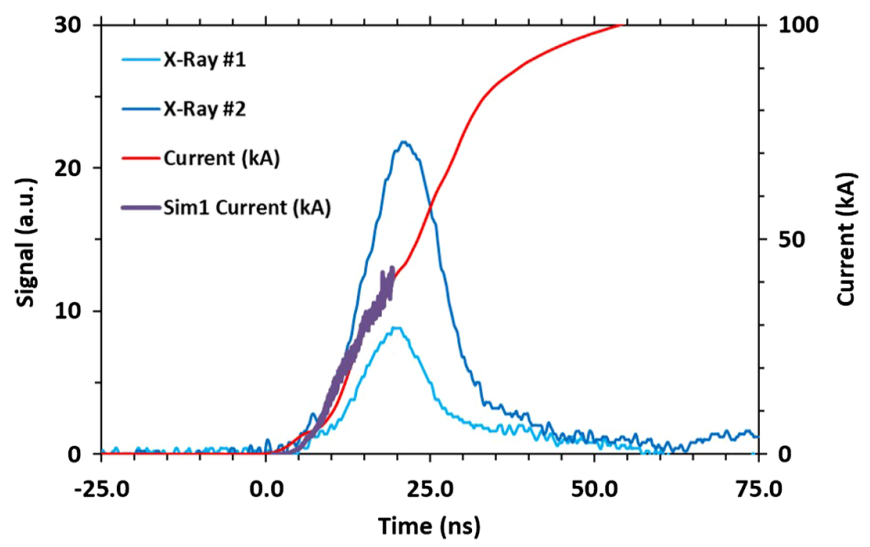

FIG. 8. The experimental current for Thunderbird shot 523 is compared with the simulated diode current $(\operatorname{sim} 1)$. The X-ray flux is also plotted from two PIN diodes near the diode. 
conditions approach $1 \mathrm{GA} / \mathrm{cm}^{2}$ current density and $200 \mathrm{TW} / \mathrm{cm}^{2}$ intensity at $10 \mu$ Torr.

We compare the simulated diode current with that of Thunderbird shot 523 (46 $\mu$ Torr vacuum pressure) derived from B-dot monitor signals [1]. The simulated current from sim1 compares well with the data as shown in Fig. 8. The $\mathrm{X}$-ray signals, created by electron impacts on the anode, are a product of both the current and voltage in the diode. Thus, we infer the voltage is falling by $20 \mathrm{~ns}$ which is also consistent with the falling simulation voltage [Fig. 4(a)]. These results suggest a vacuum pressure above $10 \mu$ Torr is compatible with good SPD operation and may be required.

\section{CHANNEL CREATION AND HOLE PROPAGATION}

An electron beam entering a material can exert a $\mathbf{J} \times \mathbf{B}$ force due to the return current flow of the ionized material plasma. If the current density is sufficient, the plasma will move outward reducing the density through which the beam moves. The beam range within the material is thus lengthened. This process can lead to hole boring even within a solid density metal. The one-dimensional equation of motion of the material can be approximated by

$$
m n \frac{d v}{d t}=J \times B,
$$

with ion mass $m$ and density $n$. In units normalized by the plasma frequency,

$$
J=\frac{4 \nu}{r^{2}} \quad \text { and } \quad B=\frac{2 \nu}{r},
$$

where $\nu$ is Budker's number (current in units of $17000 \mathrm{~A}$ ). We can then solve for the time, $t_{\exp }$, for the channel to expand 1 beam radius assuming constant acceleration,

$$
t_{\exp }=\frac{m r^{2}}{2 \nu} \frac{\omega_{p i}}{c} .
$$

The approximate rate of hole boring then is the energy dependent range $R$ in the solid material divided by the expansion time or $v=R / t_{\exp }$. This suggests the higher energy and current density produce faster penetration times.

We now inject a characterized beam into a solid $\mathrm{Cu}$ wire in a hybrid simulation. The electron beam is treated as a kinetic species and the $\mathrm{Cu}$ wire uses a quasineutral twotemperature equation-of-motion and single-group radiation diffusion described in Ref. [10]. In the SPD, we do not expect much deviation from two-dimensional behavior once the cathode plasma reaches the anode since it resembles a planar diode at this point. The beam is injected with a constant $265-\mathrm{keV}$ energy and $10-\mu$ m radius, which is $2 \mathrm{x}$ larger than that calculated in the two-dimensional

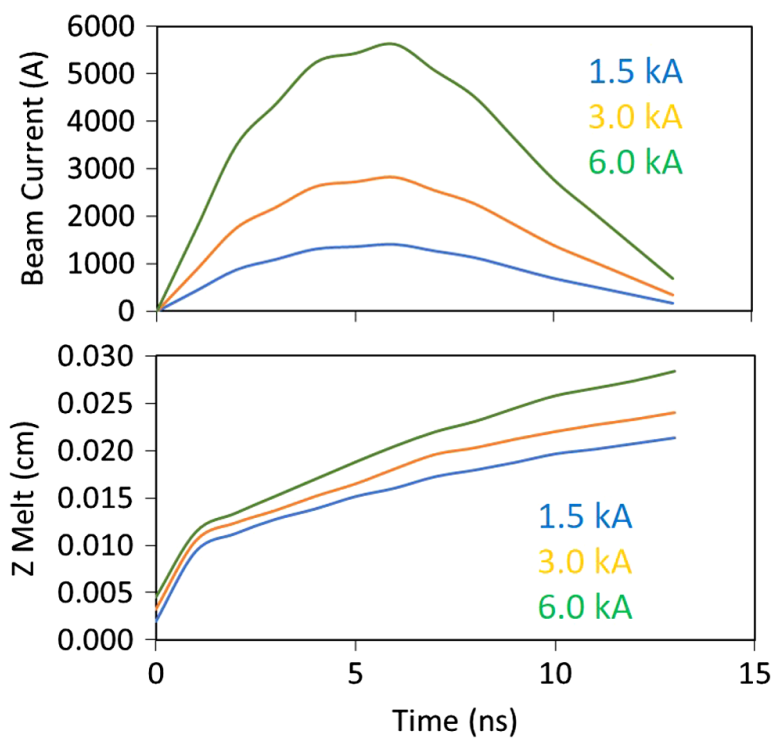

FIG. 9. The injected beam current vs time (above) and maximum position of the $\mathrm{Cu}$ melt (below) are plotted for the three hole-boring simulations with peak currents of $1.5 \mathrm{kA}$, $3.0 \mathrm{kA}$, and $6.0 \mathrm{kA}$.

simulations, to account for some dipole motion possible in three dimensional. The beam current temporal dependence, calculated in sim1, is shown in Fig. 9. We use three current profiles: the nominal (calculated in sim1) 3-kA peak, a scaled $2 x$ higher, and $2 x$ lower current. Note, the pulse has roughly a 10 -ns full width half maximum.

These three pulses are injected into a $0.2-\mathrm{mm}$ radius $\mathrm{Cu}$ wire at $0.026-\mathrm{eV}$ initial temperature and solid density. The beam currents are below the Alfven limit in all three cases, so only partial current neutralization is expected. The charge neutralization is expected to be quite good in the $>1000 \mathrm{x}$ overdense plasma. Our measure of hole boring is the maximum $z$ extent into the $\mathrm{Cu}$ wire that exceeds melt temperature. We see that all three beams melt beyond the $0.02-\mathrm{cm}$ mean free path of the $265 \mathrm{keV}$ electrons in solid $\mathrm{Cu}$ and approach $0.03 \mathrm{~cm}$ at the end of the pulse. The calculated region of melt continues to grow at a near constant rate despite the injected beam current decline indicating thermal conduction and trapped flux within the wire is still dissipating energy further into wire. From theory, we expect the beam to eject the $\mathrm{Cu}$ material within $10-\mu \mathrm{m}$ radius in roughly $17 \mathrm{~ns}$ for the nominal current, $34 \mathrm{~ns}$ for the half current, and $8.5 \mathrm{~ns}$ for the $2 \mathrm{x}$ current beam. These values for expansion time are of the order of the 10-ns beam pulse length, so hole boring is possible but somewhat weak.

The post shot picture of a $\mathrm{Cu}$ wire anode from the Thunderbird experiment is shown in Fig. 10. We see that $>0.5-\mathrm{mm}$ of the wire has been exploded off the front with a remaining hole $0.5 \mathrm{~mm}$ in length and $0.1 \mathrm{~mm}$ in radius bored into the wire. The inferred depth of beam penetration is $0.5-1 \mathrm{~mm}$. Thus, the experiment exhibits a similar hole 


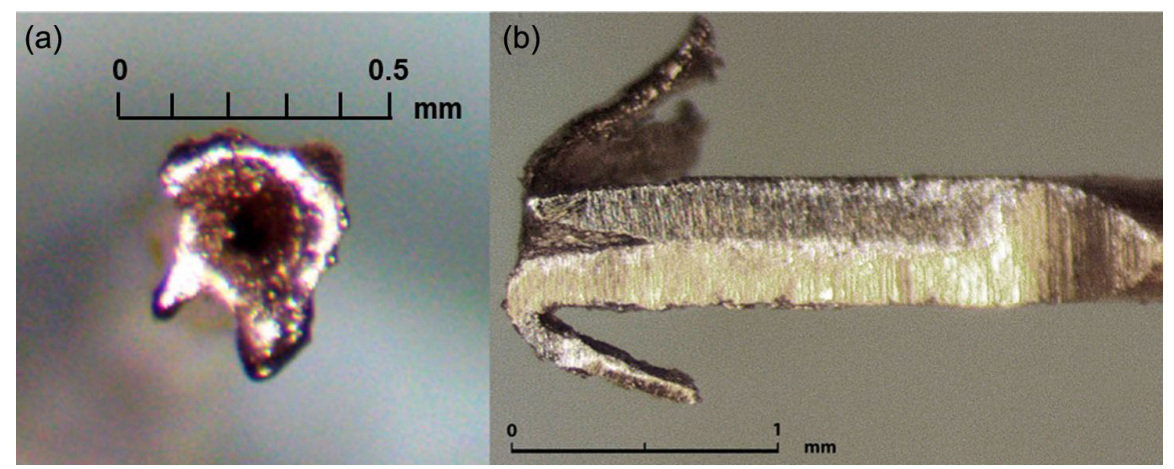

FIG. 10. A Cu wire is shown from the top (a) and bisected (b) after the Thunderbird shot. A hole has penetrated the wire up to 1 mm with $>0.5 \mathrm{~mm}$ being blown out. The width of the hole is order $0.1 \mathrm{~mm}$.

radius but longer disruption of the wire than simulated. Some of the additional hole boring beyond that seen in simulations is likely from thermal conduction and trapped flux in the wire which is apparent in the simulations after 14 ns. The electron beam and $\mathrm{Cu}$ number densities are plotted in Fig. 11 for the 3-kA peak current simulation after $14 \mathrm{~ns}$. The density has been evacuated out to $0.015 \mathrm{~cm}$ by this time allowing the electron beam to penetrate further. The electron beam density is nearly $10^{20} \mathrm{~cm}^{-3}$ at the time indicating a pile up of thermalizing electrons out to $0.023 \mathrm{~cm}$ with significant axial temperature gradient across

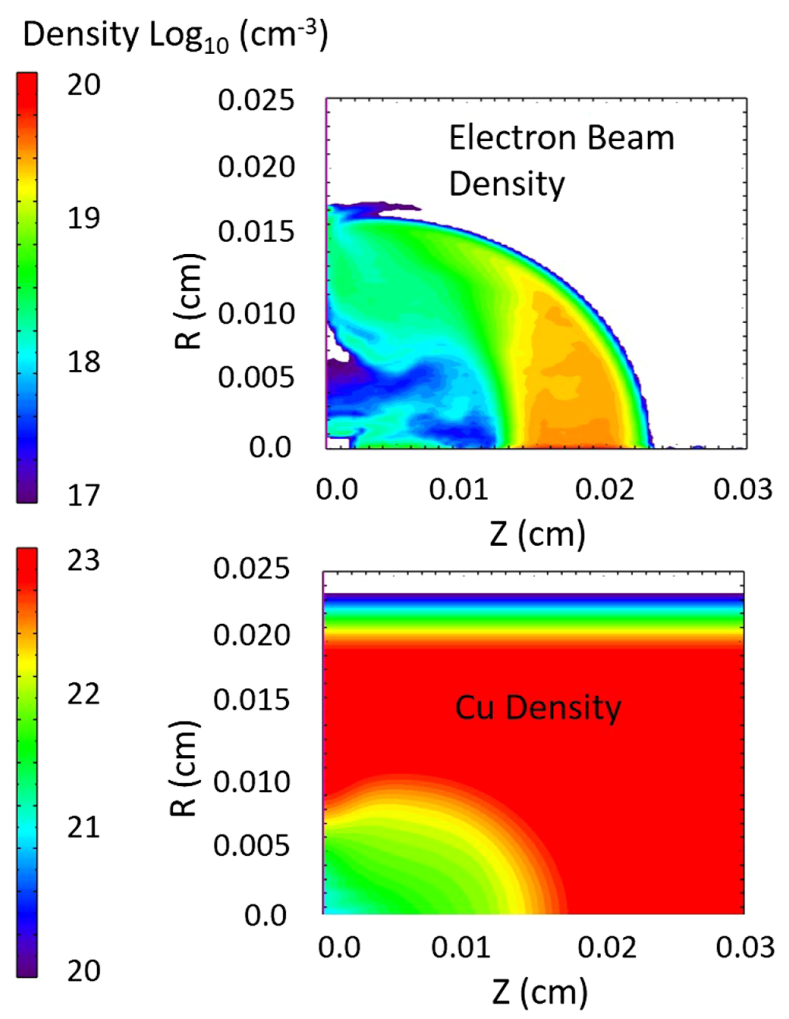

FIG. 11. The electron (top) and $\mathrm{Cu}$ (bottom) densities are plotted for the anode wire in the SPD after $14 \mathrm{~ns}$ for the 3-kA peak simulation. this region from $1 \mathrm{eV}$ to melt over roughly a $100 \mu \mathrm{m}$ distance. The resulting conduction is driving the additional melt and hole boring distance by the end of the simulations.

\section{SCALING SPD TO FUSION PLASMA APPLICATIONS}

A notional design of a reactor scale driver has been constructed and simulations of up to $9 \mathrm{MV}$ forward going voltage were completed. This design uses a simple chargedtransmission-line $10 \Omega$ circuit to fit the lower impedance diode operation. Preliminary simulations are being run with the same techniques as discussed in this paper with $10 \mu$ Torr vacuum pressure. These simulations exhibit a sustained diode voltage as high as $800 \mathrm{kV}$ for the 9-MV drive. The power is increased to $50 \mathrm{GW}$ from the Thunderbird SPD (roughly $1 \mathrm{GW}$ ). The diode operated as an SPD with deceasing impedance and increased beam intensity on axis. Details of this design and simulations are the subject for a future paper.

Initial estimates suggest that the super pinch electron beam can be used as a vehicle to deliver the energy of a pulsed-power fusion driver in a short burst through the small-radius volume of deuterium-tritium (DT) fuel, bringing it to fusion conditions and igniting the fuel at larger radius through fusion product heating. This is due to the mechanism of the energy transfer from the beam to a solidstate medium during the hole-boring process discussed in this paper. The energy of the beam of electrons is coupled with the medium as a result of electron thermalization via collisional mechanisms. Depending on the fuel density, the beam can hole bore within the fuel as it does in the metal in which case only the beam front is depositing its energy directly. Because of the structural complexity of the energy deposition region (strong anisotropy, shock generation, strong gradients of density, temperature, and electromagnetic field), an in depth understanding of this potential fusion concept requires more research.

The efficiency of ignition using the super pinch electron beam vs the DT fuel density in a small radius region is illustrated in Fig. 12. Fast thermalization of the beam 


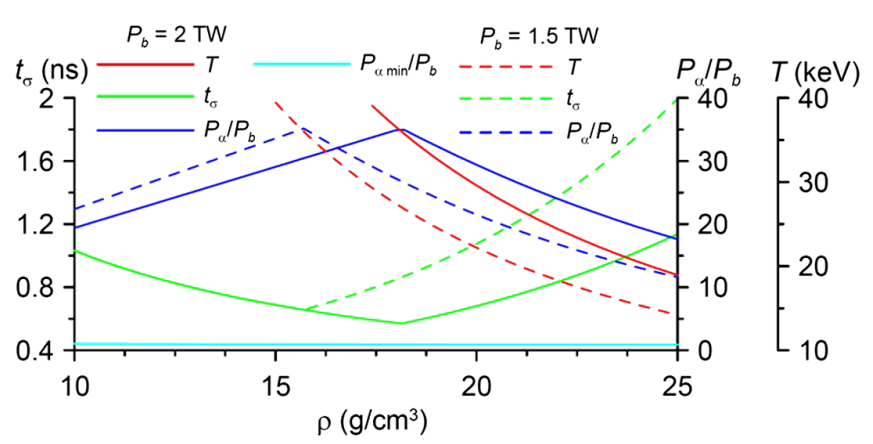

FIG. 12. The ignition efficiency of the electron beam energy absorption as a function of fuel density in the heated region at two values of electron beam power $P_{b}$. The calculated plasma temperature $T$, fusion burn time $t_{\sigma}$, and the power flux of high energy alpha-particles $P_{\alpha}$, normalized by the electron beam power, produced in the heated fuel region are plotted. Minimal value of normalized alpha-particle power flux $P_{\alpha \min }$ required to ignite the adjacent radial layer of fusion fuel (secondary ignition) is given for reference.

electrons is considered to be the major plasma-heating mechanism. Radiation cooling limits the plasma temperature rise due to beam heating to $T$. The $10-\mu \mathrm{m}$ radius of the energy-deposition region corresponds to the spatial confinement of the focused electron beam. The axial extension of this region corresponds to the scale of the collisional deceleration of the beam electrons possibly enhanced by filamentary instabilities. For $10-\mathrm{MeV}$ electrons, the standard electron range (cold $\mathrm{Cu}$ target with $8.96 \mathrm{gm}_{-\mathrm{cm}}^{-3}$ density) is $7 \mathrm{~mm}$. Here, we consider a spherical configuration and assume a $200(8.96 / \rho) \mu \mathrm{m}$ range which scales inversely with density, $\rho$. For longer range, the beam intensity must be linearly increased to obtain the same ignition results. For the same impedance operation as that observed and calculated for Thunderbird, we expect beam pinch currents approaching $100 \mathrm{kA}$ at $10 \mathrm{MV}$ voltage. In the fuel density range presented, both the thermalization time and the fuel-heating time have a similar value of approximately $1 \mathrm{~ns}$. Deposition of the electron-beam energy into lower-density fuel is not efficient because of the decreased efficiency of the energy-coupling mechanism resulting in a higher volume of the energy deposition region, while fuel "overheating" ( $T>36 \mathrm{keV})$ does not result in a faster thermonuclear burn due to the crosssection saturation. In a higher density fuel, much stronger radiation cooling prevents reaching the optimal fusion temperature resulting in slower thermonuclear burn and less efficient production of high-energy $(3.5 \mathrm{MeV})$ alphaparticle power flux $P_{\alpha}$.

According to these calculations, the optimal fusion burn conditions in a small-radius region using the super pinch electron beam are achieved at the beam power $P_{b}=2 \mathrm{TW}$ and fuel density $\rho=19 \mathrm{~g} / \mathrm{cm}^{3}$, assuming that the optimal fuel temperature is reached. This density is 2 times the density of the wire which will slow the hole boring process and lead to better coupling of beam energy to the fuel. The beam power can be reached by already existing pulsedpower technology and the fuel density can be achieved, for example, using a spherical or a cylindrical liner compression. Using a cylindrical liner compression has been shown to increase the fuel density 100 times (ten-fold radial compression) without significant instability modes (see Ref. [24]), which suggests the upper-density limit for cylindrical compression is about $23-25 \mathrm{~g} / \mathrm{cm}^{3}$. The estimated capacity of the energy storage needed for this fusion scheme is on the order of $200 \mathrm{~kJ}$.

The initial projected cost of a pulsed-power generator capable of delivering such conditions is on the order of \$20 million, which would make it one of the most costefficient, modern-day fusion experiments. Other ICF schemes present significant engineering challenges with either very expensive and high-maintenance laser systems or very-high-amplitude voltage ( $>30 \mathrm{MV}$ ) pulsed power systems, which are still beyond modern technological capabilities. The advancement of the super pinch electron beam approach could accelerate the process by utilizing already existing technology.

While the above estimations are encouraging, significantly more research is needed to complete development of the super pinch electron beam fusion process and device. For example, the focusing of the beam needs to be studied on scaled up devices. Even at this early stage, we find the possibility of a cost efficient ICF fusion scheme using the new approach strong enough to be a major motivating factor moving forward. Most importantly at this stage in the project development, we find the properties and evolution of the super pinched electron beam fully amenable to high resolution characterization, both in the diode gap and the solid anode target using the Chicago code.

\section{CONCLUSIONS}

We have studied the evolution of the electron beam generated from the SPD from experimental results of the Thunderbird accelerator and from hybrid particle-in-cell modeling and concluded that an extreme beam intensity on the anode can be generated by this type of diode. The operation is enabled by the plastic sleeve on the cathode surface with the hole on axis. While hole boring with the SPD beam has been demonstrated experimentally, the simulations have led to significant understanding of the diode physics. Our simulations show the plasma forms when the inner surface flashes over and/or experiences stimulated desorption, liberating material on the inner plastic surface. This configuration acts as a PG which shoots plasma axially toward the anode with a geometrically compressed density near the axis. The plasma connects to the cathode near the plastic interface and eventually approaches close to the anode surface. An electron beam accelerates across this sheath striking the anode. In the ideal operation, the cathode plasma stagnates its axial motion 
leaving a strong sheath only near the axis of the anode wire. At this point, the cathode plasma would be eroding ions as fast as they are resupplied at the plastic or cathode surface. Too little inventory of plasma reduces the impedance fall and limits the pinching behavior. We find a surface inventory of $8 \times 10^{15} \mathrm{~cm}^{-2}$ is sufficient for a strong pinch, but an inventory of $10^{15} \mathrm{~cm}^{-2}$ is insufficient. Beam intensities $>100 \mathrm{TW} / \mathrm{cm}^{2}$ are calculated within a $<10-\mu \mathrm{m}$ radius. Note, these intensities are calculated from twodimensional simulations and could be reduced when considering three-dimensional effects, however, we expect these three-dimensional effects to be small for low-impedance operation when the cathode plasma approaches the anode.

The characterized intense beam, with twice the radius calculated in the simulation to account for threedimensional effects, was injected into a $\mathrm{Cu}$ wire in hybrid simulations and exhibited hole boring from the $\mathbf{J} \times \mathbf{B}$ radial acceleration of the $\mathrm{Cu}$ plasma. The depth of $\mathrm{Cu}$ melted in the simulations approached $0.03 \mathrm{~cm}$ with roughly a $0.01 \mathrm{~cm}$ radius. Three peak currents were simulated showing less than linear improvement in hole boring with current. These results were somewhat less than deduced from examination of the $\mathrm{Cu}$ anode post shot, which exhibited loss of inner $\mathrm{Cu}$ out to at least $1 \mathrm{~mm}$. This would suggest a greater fraction of the diode current flows on axis than simulated, higher diode voltage than calculated, or the hole continues to form due to thermal conduction and trapped flux for a longer time than simulated. The discrepancy between simulation and experiment in the depth of hole boring is a subject for further study.

The high-beam intensity achieved with the SPD make it attractive for rapid heating of a compressed fusion fuel. The ability to transport the beam from the diode to the fuel within a metal wire offers flexibility in the fuel compression technique. Application of a scaled 10-MV, 100-kA SPD accelerator to fuel heating and ignition has been explored here, but requires considerably more research to understand the beam-fuel coupling.

[1] M. E. Weller, A. A. Esaulov, R. Presura, and D. V. Novikov, Compact differential B-dot monitors for measuring current on a fast pulsed power generator, Rev. Sci. Instrum. 90, 105102 (2019).

[2] J. R. Threadgold, I. Crotch, D. V. Rose, and J. Maenchen, 2-D LSP simulations of the self magnetic pinch diode, in Proceedings of the 14th IEEE International Pulsed Power Conference (IEEE, New York, 2003), p. 995.

[3] S. B. Swanekamp, G. Cooperstein, J. W. Schumer, D. Mosher, F. C. Young, P. F. Ottinger, and R. J Commisso, Evaluation of self-magnetically pinched diodes up to $10 \mathrm{MV}$ as high-resolution ash X-ray sources, IEEE Trans. Plasma Sci. 32, 2004 (2004).

[4] D. D. Hinshelwood, G. Cooperstein, D. Mosher, D. M. Ponce, S. D. Strasburg, S. B. Swanekamp, S. J. Stephanakis,
B. V. Weber, F. C. Young, A. Critchley, I. Crotch, and J. R. Threadgold, Characterization of a self-magnetic-pinched diode, IEEE Trans. Plasma Sci. 33, 696 (2005).

[5] K. D. Hahn, N. Bruner, S. Cordova, R. Gignac, M. D. Johnston, J. J. Lechbee, I. Molina, B. V. Oliver, S. Portillo, J. Threadgold, T. J. Webb, D. R. Welch, and D. Ziska, Overview of self-magnetically pinched-diode investigations on RITS-6, IEEE Trans. Plasma Sci. 38, 2652 (2010).

[6] S. B. Swanekamp, S. J. Stephanakis, J. M. Grossmann, B. V. Weber, J. C. Kellogg, P. F. Ottinger, and G. Cooperstein, Charged particle flow in plasma-filled pinched-electronbeam diodes, J. Appl. Phys. 74, 2274 (1993).

[7] S. B. Swanekamp, R. J. Commisso, G. Cooperstein, P. F. Ottinger, and J. W. Schumer, Particle-in-cell simulations of high-power cylindrical electron beam diodes, Phys. Plasmas 7, 5214 (2000).

[8] B. V. Oliver, K. D. Hahn, M. D. Johnston, and S. Portillo, Advances in high intensity e-beam diode development for ash x-ray radiography, Acta Phys. Pol. A 115, 1044 (2009).

[9] N. Bruner, D. R. Welch, K. D. Hahn, and B. V. Oliver, Anode plasma dynamics in the self-magnetic-pinch diode, Phys. Rev. ST Accel. Beams 14 (2), 024401 (2011).

[10] C. Thoma, C. L. Miller, D. R. Welch, N. Bennett, M. D. Johnston, B. V. Oliver, and M. L. Kiefer, Simulations of self-magnetic-pinch diode with electron and photon target transport, Technical Report No. VSL-1310, Voss Scientific, LLC, 2013.

[11] C. A. Kapetanakos and D. A. Hammer, Plasma Heating by an intense relativistic electron beam, Appl. Phys. Lett. 23, 17 (1973).

[12] G. Yonas, J. W. Poukey, K. R. Prestwich, J. R. Freeman, A. J. Toepfer, and M. J. Clauser, Electron beam focusing and application to pulsed fusion, Nucl. Fusion 14, 731 (1974).

[13] D. Prono, B. Ecker, N. Bergstrom, and J. Benford, PlasmaReturn-Current Heating by Relativistic Electron Beams with $\nu / \gamma \sim 10$, Phys. Rev. Lett. 35, 438 (1075).

[14] D. A. Hammer, K. A. Gerber, W. F. Dove, G. C. Goldenbaum, B. G. Logan, K. Papdopoulos, and A. W. Ali, Intense relativistic electron beam interaction with a cool theta pinch plasma, Phys. Fluids 21, 483 (1978).

[15] M. A. Greenspan, C. Ekdahl, J. D. Sethian, and C. B. Wharton, Plasma heating by an intense relativistic electron beam: An experimental study, Phys. Fluids 23, 205 (1980).

[16] N. Bennett, D. R. Welch, T. J. Webb, M. G. Mazarakis, M. L. Kiefer, M. D. Crain, D. W. Droemer, R. E. Gignac, M. D. Johnson, J. L. Leckbee, I. Molina, D. Nielsen, R. Obregon, T. Romero, S. Simpson, C. C. Smith, F. L. Wilkins, and D. Ziska, Phys. Plasmas 22, 033113 (2015).

[17] C. Thoma, D. R. Welch, R. E. Clark, D. V. Rose, and I. E. Golovkin, Phys. Plasmas 24, 062707 (2017).

[18] H. E. Knoepfel, Pulsed High Magnetic Fields (John Wiley \& Sons, New York, 1997), ISBN: 9780471885320.

[19] D. R. Welch, N. Bennett, T. C. Genoni, D. V. Rose, C. Thoma, C. Miller, and W. A. Stygar, Electrode contaminant plasma effects in $10^{7}-\mathrm{A} \mathrm{Z}$ pinch accelerators, Phys. Rev. Accel. Beams 22, 070401 (2019).

[20] D. R. Welch, N. Bennett, T. C. Genoni, C. Thoma, and D. V. Rose, Fast hybrid particle-in-cell technique for pulsed-power accelerators, Phys. Rev. Accel. Beams 23, 110401 (2020). 
[21] M. Li and H. F. Dylla, J. Vac. Sci. Technol. A 11, 1702 (1993).

[22] H. F. Dylla, D. M. Manos, and P. H. LaMarche, J. Vac. Sci. Technol. A 11, 2623 (1993).
[23] A. Comptant La Fontaine, J. Phys. D 40, 1712 (2007).

[24] S. F. Garanin, A. M. Buyko, and V. B. Yakubov, RayleighTaylor instability of high-velocity condensed-matter liners, J. Appl. Mech. Tech. Phys. 58, p. 779 (2017). 